\title{
Editorials
}

\section{Will we ever know if leukoreduction of red blood cells should be performed?}

\author{
Jeffrey L. Carson MD, ${ }^{*}$ Jesse A. Berlin sCD $†$
}

I $\mathrm{N}$ this month's Journal, Fergusson and colleagues describe a meta-analysis of clinical trials that evaluated the efficacy of leukoreduction of red blood cell (RBC) transfusion. ${ }^{1}$ The authors found that when all patients in the randomized trials were included in the meta-analysis, statistically significant benefits were not demonstrated. However, if only patients that actually received a transfusion were included in the analysis, a $40 \%$ reduction in postoperative infections was demonstrated. This report raises a number of questions. These include: 1) Do the results presented in this report add convincing evidence that leads to the conclusion that leukoreduction improves clinical outcomes? 2) Is it methodologically proper to include only patients who received transfusion in the analysis? We begin this commentary with some background information on leukoreduction and its rationale.

Universal leukoreduction is performed in Canada and many European countries. It remains voluntary in the United States. Leukoreduction involves the removal of white blood cells by use of filtration and may be performed either at the time of the preparation of RBC (prestorage) or concurrent with administration of (poststorage) RBC. There is a concomitant loss of red cells from 4 to $19 \%$ associated with filtration. ${ }^{2,3}$ During blood storage, white blood cells produce cytokines, which are involved with intracellular communication and influence development and maintenance of immunity and the inflammatory processes. Because these cytokines may interfere with immune function, ${ }^{4-7}$ in theory, prestorage should be superior to poststorage leukoreduction, although this has not been confirmed in clinical trials.
Allogeneic transfusion has been thought to alter immune function for many years. Allogeneic blood transfusion has been demonstrated to promote tumour growth in experimental animals. ${ }^{8,9}$ Renal allograft survival was better in patients receiving allogeneic blood transfusion prior to transplant ${ }^{3,10}$ which is consistent with a reduction in immune function. The risk of recurrent cancer has not been demonstrated in randomized control trials. ${ }^{11}$ However, it remains very controversial whether allogeneic blood transfusion increases the risk of bacterial infection.

Ten randomized clinical trials have evaluated the effects of leukoreduction. ${ }^{1}$ Scanning the results of clinical trials displayed in the table and figures in the article by Fergusson et al. reveals significant heterogeneity in the design and results of the studies. The majority of studies were performed in patients undergoing colorectal or cardiac surgery and used prestorage leukodepletion. Buffy-coated depleted blood was the standard RBC preparation used in many studies, which may make it more difficult to demonstrate a difference, since about $75 \%$ of leukocytes are removed by this process. Most of the individual studies did not demonstrate an improvement in outcome. In two of the three studies that showed differences, the observed effects were much larger than seems biologically plausible. ${ }^{12,13}$ One trial found a reduction in mortality associated with leukoreduction, although infection rate was not significantly improved. ${ }^{14}$ Thus, a meta-analysis is needed.

This meta-analysis introduces a new concept into the controversy of the efficacy of leukoreduction. Fergusson and colleagues stratify the results by whether or not the patient received a transfusion. In the analysis

From the Division of General Internal Medicine, Department of Medicine, University of Medicine and Dentistry of New Jersey, Robert Wood Johnson Medical School, ${ }^{*}$ New Brunswick, New Jersey; and the Center for Clinical Epidemiology and Biostatistics, Department of Biostatistics and Epidemiology, University of Pennsylvania School of Medicine, $\uparrow$ Philadelphia, Pennsylvania, USA.

Address correspondence to: Dr. Jeffrey L. Carson, Division of General Internal Medicine, Department of Medicine, University of Medicine and Dentistry of New Jersey, Robert Wood Johnson Medical School, 125 Paterson Street, New Brunswick, New Jersey 08903, USA. Phone: 732-235-7122; Fax: 732-235-7144; E-mail: carson@umdnj.edu

Supported in part by grant from the National Heart Lung and Blood Institute 1 R01 HL73958-01. 
of only patients who were transfused, leukoreduction was associated with lower risk of infection, although mortality was not significantly reduced (relative risk 0.61 ; $95 \%$ confidence interval $0.36-1.04$ ). The authors argue that it is proper to exclude patients who did not receive the intervention since keeping patients who did not receive blood transfusion dilutes out the effect of the treatment. While this dilution of effect is clearly a concern, it is also possible that the patients that remain in the analysis are different from those excluded. Furthermore, the advantage of randomization could be lost since it is not possible to determine if the selection of patients for transfusion by the treating physicians was made in the same way in the two treatment groups. Thus, giving up the intention-to-treat analysis potentially creates bias. Unfortunately, the data do not permit a complete exploration of this potential problem in all the studies. However, the fact that only some studies report analyses limited to those actually receiving a transfusion raises the possibility that results within this subgroup may only have been reported when they favoured leukoreduction. This is a subtle form of publication bias that may complicate the interpretation of the meta-analysis.

Our opinion is that it remains unproven that leukoreduction improves mortality or morbidity. This article provides an additional insight into the reasons why it has been difficult to consistently demonstrate a beneficial effect of leukoreduction. Future trials should only randomize patients after a decision is made to transfuse. However, we doubt a definitive leukoreduction trial will be performed in North America or Europe. Many countries, including Canada, have already implemented universal leukoreduction. Even in the US where universal leukoreduction is not required, it would be very difficult to carry out this trial since so many centres already selectively use leukoreduced blood. Perhaps centres in other parts of the world could mount this trial, although it would need to be very large to detect what is likely to be a small-moderate (10 to $20 \%$ ) improvement in outcome.

One might argue, though, that further trials are not necessary in Western countries and it is time to implement universal leukoreduction. Several reasons support this conclusion: 1) There are some data suggesting benefit; ${ }^{1,15,16}$ 2) Risks associated with leukoreduction are extremely low; 3) There is no apparent benefit of transfusing the white cells that are filtered; 4) The threshold for implementing new blood policies that might improve the blood supply has historically been very low. Recent examples include deferral of US donors who lived in the UK during the time of mad cow disease even though variant Creutzfeldt-Jacob disease has only been demon- strated to be transmitted by blood transfusion in one patient. ${ }^{17}$ Another example is nucleic acid testing which, at great cost, has been shown to detect only very few cases of human immunodeficiency viruses and hepatitis $\mathrm{C}^{18}$ 5) Leukoreduction reduces febrile non-hemolytic reactions, ${ }^{19-22}$ primary alloimmunization to human leukocyte antigens, primary transmission of cytomegalovirus, and possibly bacterial infections. Two arguments against leukoreduction are that there is a loss of red cells from 4 to $19 \%$ and cost of about $\$ 35$ US per unit. Given Western Society's sentiment to implement any blood policy that might improve safety, ${ }^{23}$ we recommend universal adoption even if we are not certain of its benefits. In other parts of the world where the cost is burdensome, further studies are needed.

\section{Saurons-nous un jour s'il faut procéder à la réduction leucocytaire des culots globulaires ?}

Dans le présent numéro du Journal, Fergusson et ses collègues présentent une méta-analyse d'essais cliniques qui évaluent l'efficacité de la réduction leucocytaire des transfusions de globules rouges (GR). ${ }^{1}$ Les auteurs ont découvert que l'inclusion de tous les patients des essais randomisés dans la méta-analyse ne permet pas de démontrer des avantages statistiquement significatifs. Par contre, l'inclusion des seuls patients effectivement transfusés produit une réduction de $40 \%$ des infections postopératoires. Ce qui soulève un certain nombre de questions : 1) Les résultats présentés apportent-ils des preuves convaincantes de l'amélioration de l'évolution clinique associée à la réduction leucocytaire ? 2) Est-il indiqué d'utiliser une méthodologie qui n'inclut que les patients transfusés dans la méta-analyse ? Commençons d'abord par des informations de base sur la réduction leucocytaire et sa logique.

La réduction leucocytaire universelle est réalisée au Canada et dans de nombreux pays européens. Elle demeure optionnelle aux États-Unis. Elle comporte le retrait des globules blancs par filtration du sang et peut être réalisée soit au moment de la préparation des GR (préconservation), soit au moment de l'administration des GR (postconservation). Il y a une perte concomitante de globules rouges de 4 à $19 \%$ associée à la filtra- 
tion. ${ }^{2,3}$ Pendant la conservation, les globules blancs produisent des cytokines, lesquelles sont impliquées dans la communication intracellulaire et influencent le développement et le maintien de l'immunité et du processus inflammatoire. Théoriquement, ces cytokines peuvent nuire à la fonction immunitaire ${ }^{4-7}$ la réduction leucocytaire préconservation pourrait donc être supérieure à celle de la postconservation, quoique les essais cliniques n'aient pas confirmé cette hypothèse.

La transfusion allogénique est perçue comme pouvant altérer la fonction immunitaire pendant des années. Ainsi, on a montré que la transfusion de sang allogène favorise la croissance des tumeurs chez des animaux de laboratoire. ${ }^{89}$ La survie après une allogreffe rénale est meilleure chez les patients qui reçoivent une transfusion allogénique avant la transplantation, ${ }^{3,10}$ ce qui va dans le sens d'une réduction de la fonction immunitaire. Le risque d'un cancer récurrent n'a pas été démontré dans les essais randomisés. ${ }^{11}$ Cependant, la controverse persiste sur la possibilité que la transfusion allogénique augmente le risque d'infection bactérienne.

Dix essais cliniques randomisés ont évalué les effets de la réduction leucocytaire. ${ }^{1}$ L'examen des résultats présentés dans le tableau et les figures de l'article de Fergusson et coll. révèle une hétérogénéité significative de la méthodologie et des résultats des études. La majorité des études a été réalisée auprès de patients de chirurgie colorectale ou cardiaque et avec l'utilisation de réduction leucocytaire préconservation. L'extraction de la couche leucocytaire était la préparation normale de GB utilisée dans de nombreuses études, ce qui peut compliquer la démonstration d'une différence, puisque environ $75 \%$ des leucocytes sont enlevés par ce procédé. La plupart des études individuelles n'ont pu montrer d'amélioration des résultats. Dans deux des trois études qui montrent des différences, les effets observés étaient beaucoup plus importants que ce qui semble biologiquement plausible. ${ }^{12,13}$ Dans un essai, on a trouvé une baisse de la mortalité associée à la réduction leucocytaire, bien que le taux d'infection n'ait pas été significativement amélioré. ${ }^{14}$ C'est pourquoi une méta-analyse est nécessaire.

Cette méta-analyse ajoute un nouveau concept à la controverse sur l'efficacité de la réduction leucocytaire. Fergusson et ses collègues ont stratifié les résultats selon que le patient avait été transfusé ou pas. Dans l'analyse des patients transfusés, la réduction leucocytaire a été associée à un plus faible risque d'infection, sans baisse significative de la mortalité (risque relatif de 0,61; intervalle de confiance de $95 \% ; 0,36-1,04)$. Les auteurs soutiennent qu'il est approprié d'exclure les patients qui n'ont pas reçu de transfusion, puisque les inclure dilue l'effet du traitement. Tandis que cette dilution est certainement préoccupante, il est aussi possible que les patients qui font partie de l'analyse soient différents de ceux qui en sont exclus. De plus, l'avantage de la randomisation pourrait être perdu, car il n'est pas possible de déterminer si la sélection des patients pour la transfusion par les médecins traitants a été faite de la même manière dans les deux groupes de traitements. Par conséquent, abandonner l'analyse qui inclut tous les patients crée un biais possible. Malheureusement, les données ne permettent pas une exploration complète de ce problème potentiel dans toutes les études. Néanmoins, le fait que seulement certaines études rapportent des analyses limitées aux patients réellement transfusés soulève la possibilité que les résultats à l'intérieur de ce groupe n'aient été signalés que lorsqu'ils favorisaient la réduction leucocytaire. C'est une forme subtile de biais de publication qui pourrait compliquer l'interprétation de la méta-analyse.

Nous croyons qu'il n'a pas été prouvé que la réduction leucocytaire diminue la mortalité ou la morbidité. Cet article donne un aperçu supplémentaire sur les difficultés de démontrer de manière conséquente un effet bénéfique de la réduction leucocytaire. Dans les futurs essais, il faudra répartir les patients après qu'une décision de transfuser aura été prise. Cependant, nous doutons qu'un essai formel sur la réduction leucocytaire soit réalisé en Amérique du Nord ou en Europe. De nombreux pays, y compris le Canada, ont déjà adopté la réduction leucocytaire universelle. Même aux É-U où la réduction leucocytaire universelle n'est pas exigée, il serait très difficile de faire cet essai, puisque de nombreux centres utilisent déjà de façon sélective du sang réduit en leucocytes. Peut-être qu'ailleurs dans le monde des centres pourraient poursuivre une telle étude, quoiqu'elle nécessite un échantillon très important pour permettre de détecter une amélioration des résultats de faible à modérée (10 à $20 \%$ ).

Malgré tout, on pourrait dire que des essais supplémentaires ne sont pas nécessaires en Occident et qu'il est temps d'appliquer la réduction leucocytaire universelle. Certaines raisons militent en faveur de cette conclusion : 1) Certaines données suggèrent des avantages $;^{1,15,16} 2$ ) Les risques associés à la réduction leucocytaire sont extrêmement faibles ; 3) Il n'y a pas d'avantage apparent à transfuser des leucocytes qui sont filtrés ;4) Les valeurs seuil pour la mise en application de nouvelles mesures visant à améliorer la réserve de sang ont été historiquement très basses. Des exemples récents de cette réalité comprennent l'exclusion de donneurs américains qui ont vécu au R-U au moment de la maladie de la vache folle même si on a pu prouver que la variante de la maladie de Creutzfeldt-Jacob par transfusion sanguine n'a 
été que chez un patient. ${ }^{17}$ Un autre exemple est le test des acides nucléiques qui, à grands frais, permet de détecter très peu de cas seulement de porteurs des virus de l'immunodéficience humaine et de l'hépatite $\mathrm{C} ;^{18} 5$ ) La réduction leucocytaire diminue les réactions non hémolytiques fébriles, ${ }^{19-22}$ l'allo-immunisation primaire aux antigènes de leucocytes humains, la transmission primaire de cytomégalovirus et les infections bactériennes possibles. Deux arguments contre la réduction leucocytaire sont qu'il y a une perte de globules rouges de 4 à $19 \%$ et que le coût est d'environ 35 \$ US par unité de sang. Étant donné le désir de l'Occident d'appliquer toute nouvelle politique pouvant améliorer la sécurité, ${ }^{23}$ nous recommandons l'adoption universelle même si nous ne sommes pas certains de ses avantages. Ailleurs dans le monde, où le coût est prohibitif, d'autres études sont à faire.

\section{References}

1 Fergusson D, Khanna MP, Tinmouth A, Hebert PC. Transfusion of leukoreduced red blood cells may decrease postoperative infections: two meta-analyses of randomized clinical trials. Can J Anesth 2004; 51: 417-24.

2 Boomgaard $M N$, Gouwerok CW, Palfenier CH, et al. Pooled platelet concentrates prepared by the plateletrich-plasma method and filtered with three different filters and stored for 8 days. Vox Sang 1995; 68: 82-9.

3 Opelz G, Vanrenterghem $\Upsilon$, Kirste $G$, et al. Prospective evaluation of pretransplant blood transfusions in cadaver kidney recipients. Transplantation 1997; 63: 964-7.

4 Bordin JO, Heddle NM, Blajchman MA. Biologic effects of leukocytes present in transfused cellular blood products. Blood 1994; 84: 1703-21.

5 Brecher ME, Pineda AA, Torloni AS, et al. Prestorage leukocyte depletion: effect on leukocyte and platelet metabolites, erythrocyte lysis, metabolism, and in vivo survival. Semin Hematol 1991; 28(3 Suppl 5): 3-9.

6 Heddle NM, Soutar RL, O'Hoski PL, et al. A prospective study to determine the frequency and clinical significance of alloimmunization post-transfusion. $\mathrm{Br} \mathrm{J}$ Haematol 1995; 91: 1000-5.

7 Smith KJ, Sierra ER, Nelson EJ. Histamine, IL-1, and IL- 8 increase in packed RBCs stored for 42 days, but not in RBCs leukodepleted pre-storage. Transfusion 1993; 33: 53S.

8 Blajchman MA, Bardossy L, Carmen R, et al. Allogeneic blood transfusion-induced enhancement of tumor growth: two animal models showing amelioration by leukodepletion and passive transfer using spleen cells. Blood 1993; 81: 1880-2.

9 Bordin JO, Bardossy L, Blajchman MA. Growth enhancement of established tumors by allogeneic blood transfusion in experimental animals and its amelioration by leukodepletion: the importance of the timing of the leukodepletion. Blood 1994; 84: 344-8.

10 Opelz G, Sengar DP, Mickey MR, Terasaki PI. Effect of blood transfusions on subsequent kidney transplants. Transplantation Proceedings 1973; 5: 253-9.

11 Vamvakas EC, Blajchman MA. Universal WBC reduction: the case for and against. Transfusion 2001; 41: 691-712.

12 Jensen LS, Andersen AJ, Christiansen PM, et al. Postoperative infection and natural killer cell function following blood transfusion in patients undergoing elective colorectal surgery. Br J Surg 1992; 79: 513-6.

13 Jensen LS, Hokland M, Nielsen HJ. A randomized controlled study of the effect of bedside leucocyte depletion on the immunosuppressive effect of whole blood transfusion in patients undergoing elective colorectal surgery. Br J Surg 1996; 83: 973-7.

14 van de Watering LM, Hermans J, Houbiers JG, et al. Beneficial effects of leukocyte depletion of transfused blood on postoperative complications in patients undergoing cardiac surgery: a randomized clinical trial. Circulation 1998; 97: 562-8.

15 Fergusson D, Hebert PC, Lee SK, et al. Clinical outcomes following institution of universal leukoreduction of blood transfusions for premature infants. JAMA 2003; 289: 1950-6.

16 Hebert PC, Fergusson D, Blajchman MA, et al. Clinical outcomes following institution of the Canadian universal leukoreduction program for red blood cell transfusions. JAMA 2003; 289: 1941-9.

17 Llewelyn CA, Hewitt PE, Knight RS, et al. Possible transmission of variant Creutzfeldt-Jakob disease by blood transfusion. Lancet 2004; 363: 417-21.

18 Jackson BR, Busch MP, Stramer SL, AuBuchon JP. The cost-effectiveness of NAT for HIV, $\mathrm{HCV}$, and $\mathrm{HBV}$ in whole-blood donations. Transfusion 2003; 43: 721-9.

19 Fung MK, Rao N, Rice J, et al. Leukoreduction in the setting of open heart surgery: a prospective cohortcontrolled study. Transfusion 2004; 44: 30-5.

20 King KE, Shirey RS, Thoman SK, et al. Universal leukoreduction decreases the incidence of febrile nonhemolytic transfusion reactions to RBCs. Transfusion 2004; 44: 25-9.

21 Paglino JC, Pomper GJ, Fisch GS, et al. Reduction of febrile but not allergic reactions to RBCs and platelets after conversion to universal prestorage leukoreduction. Transfusion 2004; 44: 16-24.

22 Yazer MH, Podlosky L, Clarke G, Nabirniak SM. The effect of prestorage WBC reduction on the rates of febrile nonhemolytic transfusion reactions to platelet concentrates and RBC. Transfusion 2004; 44: 10-5.

23 Klein HG. Will blood transfusion ever be safe enough? JAMA 2000; 284: 238-40. 